Meta

Journal des traducteurs

Translators' Journal

\title{
La réception ambivalente de Orientalism d'Edward Saïd dans le monde arabe - une question de traduction ?
}

\section{Faiza El Qasem}

Volume 61, numéro 1, mai 2016

Des zones d'incertitudes en traduction

URI : https://id.erudit.org/iderudit/1036991ar

DOI : https://doi.org/10.7202/1036991ar

Aller au sommaire du numéro

Éditeur(s)

Les Presses de l’Université de Montréal

ISSN

0026-0452 (imprimé)

1492-1421 (numérique)

Découvrir la revue

Citer cet article

El Qasem, F. (2016). La réception ambivalente de Orientalism d'Edward Saïd dans le monde arabe - une question de traduction ? Meta, 61(1), 221-236. https://doi.org/10.7202/1036991ar
Résumé de l'article

Tout acte de traduction laisse des traces dans la mesure où le traducteur " emprunte " une voix qui n'est pas la sienne. Ce faisant, le traducteur révèle la clé de sa démarche, qui vise soit à s'effacer derrière la voix de l'auteur traduit, à être le passeur en quelque sorte d'une autre parole poétique, d'un autre regard sur le monde, soit au contraire à imprimer sa voix, à influer sur le traduire. Cela implique une prise en compte de la visibilité du traducteur, donc de sa subjectivité et par là même, une remise en cause des concepts traditionnels de la théorie et de la pratique de la traduction ayant trait à la fidélité, au sens et à l'interprétation. Traduire, c'est réécrire l'original non seulement pour faire passer un sens mais aussi pour refléter une certaine idéologie, introduire de nouveaux concepts, de nouveaux genres, bref, innover. La position du traducteur face à l'altérité diffère selon qu'il cherche à narguer les règles de sa langue culture estimant qu'elles ne suffisent pas à rendre compte du contenu du texte à traduire, ou à l'inverse, à ne pas laisser subsister de points d'interrogation pour ne pas désorienter le lecteur et concevoir ainsi la lecture d'un texte traduit comme si l'auteur s'était directement exprimé dans la langue de traduction.

Dans ce contexte, nous interrogerons les pratiques des traducteurs arabes de l'ouvrage phare d'Edward Saïd, Orientalism, telles qu'elles sont données à lire à travers leurs préfaces. Nous ferons ensuite le lien avec la réception de l'ouvrage dans le monde arabe. Ces préfaces interrogent aussi le statut du traducteur : auteur de plein droit ou traducteur découvreur? Telles sont les questions auxquelles nous nous intéresserons pour étudier l'empreinte du traducteur sur le texte.
Ce document est protégé par la loi sur le droit d'auteur. L'utilisation des services d'Érudit (y compris la reproduction) est assujettie à sa politique d'utilisation que vous pouvez consulter en ligne.

https://apropos.erudit.org/fr/usagers/politique-dutilisation/ 


\title{
La réception ambivalente de Orientalism d'Edward Saïd dans le monde arabe - une question de traduction?
}

\author{
FAIZA EL QASEM \\ Université Paris 3 - Sorbonne Nouvelle, Paris, France \\ f.elqasem@hotmail.fr
}

\section{RÉSUMÉ}

Tout acte de traduction laisse des traces dans la mesure où le traducteur «emprunte» une voix qui n'est pas la sienne. Ce faisant, le traducteur révèle la clé de sa démarche, qui vise soit à s'effacer derrière la voix de l'auteur traduit, à être le passeur en quelque sorte d'une autre parole poétique, d'un autre regard sur le monde, soit au contraire à imprimer sa voix, à influer sur le traduire. Cela implique une prise en compte de la visibilité du traducteur, donc de sa subjectivité et par là même, une remise en cause des concepts traditionnels de la théorie et de la pratique de la traduction ayant trait à la fidélité, au sens et à l'interprétation. Traduire, c'est réécrire l'original non seulement pour faire passer un sens mais aussi pour refléter une certaine idéologie, introduire de nouveaux concepts, de nouveaux genres, bref, innover.

La position du traducteur face à l'altérité diffère selon qu'il cherche à narguer les règles de sa langue culture estimant qu'elles ne suffisent pas à rendre compte du contenu du texte à traduire, ou à l'inverse, à ne pas laisser subsister de points d'interrogation pour ne pas désorienter le lecteur et concevoir ainsi la lecture d'un texte traduit comme si l'auteur s'était directement exprimé dans la langue de traduction.

Dans ce contexte, nous interrogerons les pratiques des traducteurs arabes de l'ouvrage phare d'Edward Saïd, Orientalism, telles qu'elles sont données à lire à travers leurs préfaces. Nous ferons ensuite le lien avec la réception de l'ouvrage dans le monde arabe. Ces préfaces interrogent aussi le statut du traducteur: auteur de plein droit ou traducteur découvreur? Telles sont les questions auxquelles nous nous intéresserons pour étudier l'empreinte du traducteur sur le texte.

\section{ABSTRACT}

Every act of translation leaves a trace insofar as the translator "borrows" a voice that is not his. In doing so, the translator reveals the key to his approach, whereby he aims either to hide behind the voice of the translated author, to be a sort of a go-between, as it were, of another's poetic expression, of a different perspective on the world, or, conversely, to imprint his voice and thus exert an influence on the translated text. This notion takes into account the visibility, and hence the subjectivity, of the translator, thereby challenging traditional concepts within the theory and practice of translation with regard to fidelity, meaning and interpretation. To translate is not only to rewrite the original with the purpose of conveying its specific meaning, but also to reflect a certain ideology, to introduce new concepts and genres, in short, to innovate.

The position of the translator in the face of alterity differs depending on if he chooses to flout the rules of his cultural language, considering it insufficient for capturing the content of the text to be translated, or, on the contrary, if he chooses to eliminate unresolved issues in order to avoid disorienting the reader, thus formulating a reading of the translated text as if the author had directly expressed himself in the target language.

Working within this framework, we will examine the practices and methodologies of Arabic translators of Edward Saïd's monumental work Orientalism as they are presented 
in the prefaces to the various translated editions. We will then establish a link with the reception of Saïd's work in the Arab world. The prefaces also question the role of translators: are they authors in their own right or translators/discoverers? These are the principal questions of interest in this study on the imprint of the translator on the text.

\title{
MOTS CLÉS/KEYWORDS
}

frontière, voix, trace, emprunt, explicitation-implicitation, naturalisation-exotisation frontier, voice, trace, borrowing, explicit-implicit, foreignization-domestication

\begin{abstract}
"Chaque époque et chaque société recrée ses propres “autres” d de même que "l'identité humaine est non seulement ni naturelle ni stable, mais résulte d'une construction intellectuelle, quand elle n'est pas inventée de toutes pièces.»
\end{abstract}

(Edward Saïd 1978/1980: 358-359)

\section{Introduction}

L'exploration de la réception des textes traduits dans une culture d'arrivée doit permettre de comprendre la fonction de ces textes et la façon dont ils s'ajustent aux normes de la traduction qui prévalent alors, entrant en contradiction ou en consonance avec elles et modifiant éventuellement le système littéraire donné. Il s'agit ici de s'intéresser à la réalité du texte traduit, à son statut: texte second ou texte à part entière? Ce statut du texte traduit peut nous amener à nous intéresser au statut du traducteur, à son projet traductif dans un contexte culturel donné, aux discours critiques tenus sur le texte.

Nous avons choisi de traiter des deux traductions arabes du célèbre essai d'Edward Saïd, Orientalism, et de leur réception dans le monde arabe, en privilégiant le point de vue des traducteurs tel qu'il est donné à lire dans les préfaces des traductions. Une différence temporelle importante sépare les deux textes, et en nous interrogeant sur leur réception, nous tiendrons compte de leurs conditions de lecture. Pourquoi précisément cet essai? En raison du faible écho qu'il a suscité dans le monde arabe au moment de sa parution alors que partout ailleurs, on s'est largement inspiré du travail de Saïd, selon les dires de Mohamed Shahine (2007: 7)

L'ouvrage suscita maintes controverses au moment de sa parution, mais permit aux études postcoloniales de se développer. En effet, l'argumentation de Saïd consistait à démontrer que le regard et l'analyse que nous portons sur «les Autres» et donc sur l'Orient ne sont jamais neutres, mais s'inscrivent dans une tradition et un discours donnés, ce que Tzvetan Todorov qualifie dans sa préface à la traduction française de l'essai de "pression des sujets parlants et interprétants sur la formation des discours» (Todorov dans Saïd 1980: 7).

Comme l'écrit Jean-Marc Moura, l'un des premiers critiques français à introduire la théorie postcoloniale dans les études francophones,

l'ouvrage d'Edward Saïd, L'Orientalisme, a donné l'exemple d'un type d'étude où les textes philosophiques, littéraires, ethnographiques occidentaux sont analysés comme les signes d'un discours politique sur l'ailleurs de l'Europe. (Moura 1999: 48)

Notre propos n'est cependant pas ici de nous focaliser sur le contenu de l'ouvrage, mais plutôt de chercher à savoir si le commentaire péritextuel se révèle comme le lieu 
de résolution de la tension entre le texte et son traducteur, s'il nous renseigne sur les enjeux d'une réception ambiguë et complexe du livre, s'il permet de mettre en avant le sujet traducteur et son habitus traductionnel, et comment tout cela laisse une trace explicite ou implicite dans le discours traduit.

Commençons par dire qu'un quart de siècle sépare très exactement les deux traductions arabes de Orientalism. Celle de Kamal Abu Deeb a été effectuée en 1981, soit trois années après la parution du livre en 1978. Entre-temps, la traduction française par Catherine Malamoud est parue au Seuil en 1980 et Kamal Abu Deeb y fait souvent référence dans sa préface.

La seconde traduction arabe, par Mohamed Anânî en 2006, se veut une traduction complète de l'édition augmentée de 1995, comme le précise d'emblée le traducteur dans sa préface. Ce qu'il justifie comme le droit pour le lecteur arabe de prendre connaissance de la forme finale du livre après les centaines d'études qui lui ont été consacrées, aussi bien en Orient qu'en Occident, que ce soit sous la forme d'études universitaires ou d'écrits scientifiques.

En somme, la retraduction de Mohamed Anânî paraît à une époque où l'œuvre originale a déjà accompli une traversée spatiale et temporelle: de nombreuses traductions du livre ont été effectuées, des articles scientifiques ont commenté l'œuvre et ont fait également apparaître le réseau dans lequel elle fait sens et se stabilise (Latour 1987: 45).

\section{Le corpus, les auteurs et la méthode}

Nous avons écrit plus haut que les deux versions ne s'appuyaient pas sur le même texte source; elles ne portent pas le même sous-titre non plus. Par comparaison, dans sa version française, Catherine Malamoud choisit elle aussi de remanier quelque peu le titre original Orientalism en lui adjoignant un sous-titre, l'Orient créé par l'Occident, que l'on retrouve parfois dans certains commentaires du livre original sous "Western conceptions of the Orient». En arabe, Kamal Abu Deeb choisit comme sous-titre: «Le discours, le pouvoir, le savoir» (al inshâ', as-sulta, al-ma'rifa), tandis que Mohamed Anânî opte pour «L'Orient, au regard de l'Occident » (al mafâhîm al gharbiyya lil sharq).

Dans la troisième de couverture de la version française, juste après la préface de Todorov, on lit:

Le texte de l'Orientalisme publié ici diffère légèrement de l'original anglais, car il a été adapté en vue du public français: quelques développements (concernant notamment la scène américaine) sont supprimés, quelques explications ajoutées. Toutes les omissions sont signalées par [...]. L’index a été limité aux seuls noms propres. (Saïd 1980: 10)

Orientalism est un texte fondateur qui a favorisé l'émergence des études postcoloniales et leur réception dans le champ académique en France et ailleurs dans le monde. Traduit dans plus d'une trentaine de langues, il s'adresse à un lecteur spécialisé. Son auteur, d'origine palestinienne, est un spécialiste de littérature comparée et s'intéresse à la théorie et à la critique littéraires. Il a enseigné de 1963 à 2003 à l'Université Columbia de New York. Yves Clavaron le présente comme « un penseur dans le monde» car la théorisation saïdienne selon lui se caractérise par "une volonté de concilier activité critique et engagement politique contre toute forme d'impérialisme» (Clavaron 2013: 35). 
Quant aux traducteurs arabes, ils jouissent d'un capital culturel certain: ils sont tous deux universitaires. Kamal Abu Deeb a occupé un temps la chaire des études d'arabe à la School of Oriental and African Studies de Londres. Il est spécialiste de poésie et de critique littéraire arabes, et outre Orientalism, a également traduit Culture and Imperialism (1993) d'Edward Saïd.

Dans la quatrième de couverture de la seconde traduction, Mohamed Anânî est présenté comme l'auteur de la traduction fine et explicite de l'ouvrage, professeur à l'Université du Caire, et traducteur chevronné ayant traduit en 2005 vers l'arabe deux ouvrages de Saïd: Representations of the Intellectual: The 1993 Reith Lectures (1994) et Covering Islam. How the Media and the Experts Determine How we See the Rest of the World (1981) (ces deux ouvrages ont également fait l'objet d'une traduction française, respectivement: Des Intellectuels et du pouvoir et L'Islam dans les médias. Comment les médias et les experts façonnent notre regard sur le reste du monde).

En tant que tels, les deux traducteurs appartiennent au même milieu professionnel: la littérature et la traduction. Il y a donc une nette relation entre leur habitus social et le choix du sous-domaine de spécialité qu'ils traduisent. Au moment où ils s'attellent à la traduction, ils occupent déjà une position d'influence dans le milieu institutionnel. Cela dit, vu le laps de temps très court qui sépare la parution de l'ouvrage de sa traduction arabe, les retombées positives du livre de Saïd rejaillissent nécessairement sur la première traduction, dont la tâche essentielle est d'introduire l'œuvre étrangère auprès d'un nouveau public, ici arabe. Tel n'est pas le cas d'une retraduction «qui est généralement plus attentive que la traduction-introduction, la traduction-acclimatation, à la lettre du texte source, à son relief linguistique et stylistique, à sa singularité », comme le dit Paul Bensimon dans sa présentation du $n^{\circ} 4$ de la revue Palimpsestes, «Retraduire» (Bensimon et Coupaye 1999: X).

Les noms des traducteurs apparaissent sur la couverture, mais l'éditeur choisit la mention (naqalahu ilal arabiyya) qui signifie "transposé en arabe par», s’apparentant davantage à la translation dans le cas d'Abu Deeb tandis que dans le cas de la traduction d’Anânî, il est inscrit «traduit par Mohamed Anânî», ce qui pourrait laisser croire à une transposition dans le premier cas. Nous verrons si cela se vérifie dans les stratégies de réécriture employées (préface, table des matières, glossaire). En tout état de cause, le traducteur, son histoire, son «profil» et sa posture traductive occupent une place essentielle dans l'analyse de l'œuvre traduite (Inghilleri 2003: 15).

\section{Typologie du texte}

Orientalism est un ouvrage de sciences humaines. Il s'agit de voir si la traduction de ce type d'ouvrages diffère de celle d'ouvrages techniques et scientifiques. Nous pensons que les deux types de textes exigent une parfaite connaissance du domaine à la fois linguistique et extra-linguistique. Cependant, les sciences humaines sont souvent contraintes par leur environnement socioculturel. En effet, le discours des sciences humaines se transmet avec des concepts qui sont partagés ou contestés par une communauté de chercheurs, qui s'apparentent à des termes techniques propres à une culture, et dont la portée conceptuelle peut varier en fonction du contexte. Ces concepts sont souvent liés à une période donnée, à un courant idéologique; ils peuvent être remis en cause ou au contraire s'ancrer définitivement. 
Le traducteur doit donc non seulement être averti du sujet dont traite le texte, mais aussi du contexte disciplinaire dans lequel s'élabore ce texte.

Salah Mejri, pour sa part, considère que la traduction des textes spécialisés tels que ceux des sciences du langage «répond à des besoins précis, [...] et justifie le transfert des connaissances encyclopédiques disponibles en L1 vers L2. Cette dissymétrie de départ engendre un grand enchevêtrement dans les problématiques posées par la dimension terminologique» (Mejri 2008: 120).

Cette dimension terminologique est mise en avant par le traducteur Abu Deeb. Il l'explique dans sa préface en termes de retard: l'un des deux partenaires (ici l'arabe) techniquement en retard sur l'autre reconnaît ce retard et la nécessité de le combler. Nous verrons plus avant la part d'incertitude dans la traduction de certains concepts, engendrée par la «migration de notions, entre les langues, entre les disciplines, ou simplement le long de l'axe temporel» (Samain 2014: 181).

\section{La voix du traducteur comme instance préfacielle}

Les deux préfaces assument clairement une fonction métadiscursive et se proposent de guider la compréhension du lecteur. Lieux d'information et/ou de recommandation concernant le texte, elles ont une fonction didactique qui invite à lire dans l'espace arabophone des recherches issues d'autres champs didactiques. Cette transmission de la connaissance dont va se charger le traducteur est orientée en fonction de sa vision propre, l'œuvre ne se donnant pas en effet au premier lecteur mais à un lecteur exercé, qui va procéder à son évaluation et réfléchir à la manière dont il l'introduira dans la culture d'arrivée. Le traducteur partage avec le lecteur la langue et ses normes, mais aussi les normes socioculturelles. Il situe l'importance de l'ouvrage traduit dans le contexte socioculturel qui l'accueille, assumant ainsi un rôle de médiateur. Et comme le dit Genette, ce passage du livre qu'est la préface, «s'il n'est pas encore le texte, il est déjà du texte» (Genette 1987: 13), puisque le traducteur se propose de donner certaines clefs au lecteur, lui permettant de comprendre le texte pour mieux appréhender la traduction qu'il lui propose et qu'il espère la plus appropriée possible à la culture d'accueil. Par ailleurs, l'étayage de la traduction dont parle Berman (1995: 68), c'està-dire l'ensemble des métatextes (notes et glossaires), vient ici soutenir la traduction et représente une importante source d'information pour apprécier les prises de décision du traducteur ainsi que les stratégies de traduction utilisées.

Dans le cas qui nous préoccupe, les préfaces commencent par contextualiser le livre et témoigner de la très grande érudition de l'auteur, mentionnant au passage les références vastes et variées sur lesquelles il s'appuie: politique, ethnologie, littérature, histoire, géographie. Elles sont très longues: 19 pages au total chez Abu Deeb, dont 10 consacrées aux problèmes de traduction, et 21 pages chez Anânî, dont 10 également consacrées à la traduction.

La préface d'Anânî donne de précieuses indications, sous forme de citations et de références précises, sur la réception des idées de Saïd dans le monde et sur des thèses universitaires qui ont été soutenues en Égypte, à l'Université du Caire entre autres, sur la réception de la pensée saïdienne dans le monde arabe, à la suite de la traduction de Orientalism.

Abu Deeb est séduit d'emblée par l'éloquence de l'auteur et par la hardiesse du propos. Il exprime sa fascination et parfois sa perplexité devant cette pensée profonde, 
exigeante, sophistiquée. Dès la première ligne, Abu Deeb fait preuve de révérence à l'égard de ce livre qu'il présente comme jouant un rôle très actif dans une nouvelle révolution de la recherche en sciences humaines, laquelle révolution plonge ses racines dans le marxisme, la révolution linguistique et structuraliste, et dans ce que l'on peut considérer comme une école nouvelle du courant historiographique baptisé «la Nouvelle Histoire», qui s'inscrit dans la filiation de Foucault.

Il émet une appréciation positive du livre qu'il traduit pour mieux justifier ses choix et ses stratégies et intervenir par la suite dans le contenu du livre pour expliciter, clarifier, voire renforcer l'argumentation originale. Le traducteur s'interroge: n'est-il pas simpliste de dire que le livre de Saïd est difficile à lire et à traduire? Relativement à cette pensée foisonnante, le problème se pose effectivement au traducteur, mais non en termes de facilité ou de difficulté. Car la difficulté est ailleurs: il s'agit d'être capable de vulgariser un message analytiquement et conceptuellement complexe. Selon lui, la difficulté de la traduction dans ce cas d'espèce n'est pas unidimensionnelle, mais multidimensionnelle.

La langue arabe semble pour lui l'objection préjudicielle en quelque sorte qui explique l'impossibilité de traduire des concepts complexes vu l'état de développement de la langue arabe au moment où il traduit. L'intraduisibilité procéderait ici d'une limite intrinsèque du langage incapable d'exprimer l'état d'avancement des connaissances ni l'évolution de la pensée contemporaine. Autrement dit, pour que la langue puisse jouer son rôle en tant qu'«acte social» répondant à un besoin de communication, il faut que celle-ci permette la création de mots nouveaux comme l'affirme Joseph Vendryes (1921: 1-2). C'est ce que Mejri appelle «l'espace terminologique où se concentrent les concepts mis en débat soit pour le caractère controversé de leurs dénominations, soit pour un aspect quelconque concernant leur statut non encore bien établi dans la métalangue» (Mejri 2008: 121).

Abu Deeb en veut pour preuve le phénomène de l'acculturation qui eut lieu au Moyen-Âge dans le contexte des traductions arabes et qui témoigne de l'extraordinaire audace des traducteurs en matière de créativité expressive et de néologismes, lesquels n'hésitaient pas à entraver parfois les structures rigides de la langue. Ce sont, en effet, les traducteurs arabes, protecteurs et propagateurs des cultures, qui permirent à la langue arabe d'évoluer et de devenir instrument de culture. Le lieu du traducteur-philosophe était un lieu de tension et de création sur le plan conceptuel et linguistique.

Par cette allusion-suggestion aux traducteurs du Moyen-Âge, Abu Deeb réussit un coup double: celui de se hisser au même niveau que ses illustres prédécesseurs et celui d'anticiper les éventuels partis pris contre sa traduction audacieuse susceptibles de susciter les mêmes débats et controverses dont furent l'objet les traductions philosophiques arabes de la part des grammairiens. Il livre alors sa conception de la traduction en partant d'une analyse du livre de Saïd: à ses yeux, le texte représenté est l'incarnation d'une pensée, d'une vision du monde, et d'une manière particulière d'user de la langue. Autrement dit, la pensée créatrice de Saïd s'incarne dans la matière de la langue. Aussi, la tâche du traducteur devrait être la représentation d'une double efficience: l'incarnation d'un texte dans une langue cible élargie afin qu'elle puisse exprimer l'inédit: 
Une langue élargie implique l'implosion de la langue sans laquelle on serait contraint d'exprimer toute chose en recourant à l'explicitation, à la simplification et au discours direct. Cette langue dilatée, élargie, serait alors riche en potentialités et plus souple. (Abu Deeb dans Saïd 1981: 16; notre traduction)

Ne plus exercer cette audace envers la langue équivaudrait ni plus ni moins à ne plus nourrir les esprits par la fréquentation des œuvres, voire à empêcher l'évolution de la pensée. Cette idée de dilatation se rapproche du concept de l'élargissement de la langue ou encore de l'acceptation de l'Étranger dans la langue chère à Berman (1984: 49).

Abu Deeb convoque plusieurs auteurs pour justifier sa posture mais nous donne tout d'abord sa conception de la traduction qui se réduit à une double dimension: la représentation du texte traduit de façon à bien saisir ses caractéristiques macrostructurales et l'expression de cette représentation en une langue capable d'incarner au mieux ces caractéristiques:

$\mathrm{Si}$, jusque-là, la traduction consistait à couler la compréhension d'un texte dans le moule préétabli de la langue, il n'en est plus de même aujourd'hui. En effet, traduire, c'est reproduire la pensée dans la matérialité de la forme. (Abu Deeb dans Saïd 1981: 17; notre traduction).

Ce que j'essaie de faire ici dépasse la simple transposition du texte sur le plan sémantique puisque je me heurte, dans le contexte actuel de la langue et de la culture arabes, à des problèmes épineux liés aux potentialités de la langue à représenter le monde et par là même, à transférer une œuvre dans une autre communauté culturelle. D'où la nécessité pour le traducteur de recourir à tous les moyens qui lui permettront d'enrichir la langue, même si d'aucuns devaient les qualifier de transgressifs par rapport au discours arabe, au style arabe «familier». (Abu Deeb dans Saïd 1981: 18; notre traduction)

La grande part d'incertitude dans la traduction d'Abu Deeb est liée précisément à sa conception de la traduction et se reflète dans divers décalages: énonciatifs, sémantiques, syntaxiques. Il assume ses choix parce qu'il pense qu'en bousculant les règles de la langue, en y introduisant une part d'étrangeté, on l'aide à s'épanouir, à s'ouvrir sur l'Étranger. Le lecteur n'est pas pris en compte ici, il doit se contenter du glossaire de 13 pages mis à sa disposition pour comprendre les créations audacieuses du traducteur. Cette question centrale se résume en traduction, comme le rappelle Antoine Berman dans L'épreuve de l'étranger, par le fameux paradoxe posé par Schleiermacher: «amener le lecteur à l'auteur» ou "amener l'auteur au lecteur» (Berman 1984: 15).

En bien des endroits, Abu Deeb fait fi de la compréhension du lecteur en ne dissociant pas assez les idiomes en présence. Or, comme l'explique Fortunato Israël:

plus la matérialité de la forme est importante [...], moins elle est reproductible, et plus il devient nécessaire de dissocier les idiomes pour retrouver, par d'autres biais, la même charge émotive. (Israël 1994: 109)

Abu Deeb justifie sa démarche sur le plan terminologique en invoquant l'imprécision des termes en matière de critique littéraire, de politique, de sociologie, de sciences. Que dire alors des termes nés d'écoles ou de disciplines nouvelles européennes dont l'usage est restreint à quelques locuteurs et pour lesquels d'autres correspondants existent pourtant déjà dans la langue arabe, obstruant ainsi la compréhension et l'harmonisation terminologique? Le traducteur expose ses choix: 
J'ai utilisé des formulations, des termes et des constructions qui sont globalement assez clairs pour le lecteur arabe dans certains cas. D'autres revêtent une signification qui diffère de celle dont ils ont l'habitude. D'autres n'ont pas de signification arrêtée en langue arabe. Mais je les ai choisis en toute conscience parce qu'ils étaient conformes à ma conception de la traduction. Je les ai regroupés dans un glossaire pour faciliter leur compréhension en contexte et pour qu'il y ait une interaction permanente entre la langue de la traduction et la langue de l'original. (Abu Deeb dans Saïd 1981: 18; notre traduction)

Abu Deeb met l'accent sur l'apparition de nouveaux concepts nés de l'évolution de la connaissance; il ressent le besoin de dénommer la nouvelle réalité, et procède soit en investissant une forme existante d'un nouveau sens, soit en imposant une forme nouvelle à un sens ancien.

Or, dans son intention de «dilater» la langue, il ne fait ni plus ni moins qu'opacifier des concepts. Le lecteur arabe est heurté par la complexité du lexique proposé, par la verbosité de la forme, alors même que le traducteur explique par ailleurs que le problème terminologique n'est pas le seul qu'affronte le traducteur arabe, mais qu'il en est un autre qui réside dans la capacité de la langue à traduire un texte avec précision, concision et fluidité sans le paraphraser ou l'expliciter et sans constamment changer les termes utilisés pour traduire les notions étrangères. Autrement dit, peuton traduire un terme étranger directement par un terme arabe partageant les mêmes caractéristiques et le même réseau sémantique et utiliser par la suite ce même terme arabe dans tous les contextes où il apparaît?

Abu Deeb fait allusion ici aux termes n’ayant pas bénéficié de l'assise nécessaire à une intégration totale. Selon Mejri, il s'agit dans la plupart des cas d'innovations d'auteurs qui n'ont jamais été reprises par les autres spécialistes et qui collent aux textes dans lesquels elles ont pris naissance (2008: 123).

Le pire cependant est que le traducteur se soit permis d'utiliser des termes de la tradition littéraire arabe classique en lieu et place des termes arabisés de longue date et entrés dans l'usage, comme discours, mimesis, modèle ou code. Ce faisant, il crée des décalages sémantiques et met en place de faux amis conceptuels puisés dans deux traditions culturelles différentes, faussant le sens voulu par l'auteur et augmentant la part d'incertitude dans l'interprétation des notions:

The main achievement of Abu Deeb's painstaking translation was an almost total avoidance of Arabized Western expressions; technical words like discourse, simulacrum, paradigm, or code were rendered from within the classical rhetoric of the Arab tradition. His idea was to place my work inside one fully formed tradition, as if it were addressing another from the perspective of cultural adequacy and equality. (Saïd 1978/2003: 339)

La tradition rhétorique est tellement prégnante que le recours à certains des termes de son stock imposé par la traduction conduit systématiquement à des confusions qui risquent de nuire au texte cible. Prenons l'exemple du terme discours pour lequel Abu Deeb propose inshâ', sous prétexte qu'il se prête à la dérivation; or, ce dernier signifie l'art de rédiger et son sens étymologique désigne la création. Abu Deeb confère ainsi un sens nouveau à un terme connu et le préfère au terme khitâb, signifiant pourtant habituellement discours. Et que penser de l'usage de al ishâriyya, néologisme créé de toutes pièces et utilisé de nos jours en tant qu'adjectif dans la 
locution lugha ishariyya signifiant langue des signes, en lieu et place de al dalâla, terme consacré pour dire signification?

Enfin, deux exemples de faux amis conceptuels: le terme orthodoxy est rendu par sunnisme, al sunniya, chez Abu Deeb, qui le justifie ainsi: «vision conservatrice au sens général et non en rapport avec le courant religieux en islam». Anânî choisit de dire "courant conservateur», al tayyâr al muhâfiz. De même, le simple terme de théologie, cité ici en tant que discipline, a été traduit par charia au lieu de lâhût, connu du lecteur arabe: «Besides, a field can change so entirely, in even the most traditional disciplines like philology, history or theology, as to make an all-purpose definition of subject matter almost impossible» (Saïd 1995: 50).

Autre part d'incertitude: les décalages de type syntaxique. Le traducteur assume là aussi des choix syntaxiques qui transgressent, dit-il, les règles de l'usage (Abu Deeb dans Saïd 1981: 19; notre traduction). Il calque ainsi une structure anglaise qui consiste à utiliser deux prépositions se rapportant à une même annexion du type: "his contribution to and development of the field" sous prétexte qu'elle est plus concise, faisant fi des relations hiérarchiques que les unités entretiennent entre elles. Ailleurs, il décide de coordonner deux substantifs ayant le même complément de nom du type: «le maître et les disciples de cette École» (Abu Deeb 1981: 16) alors qu'il faut les séparer en arabe: (صاحب وتلاميذ هذه المدرسة الفكرية) puisqu'on ne peut en principe rien intercaler entre le nom et son complément de nom. L'usage voudrait qu’on dise: «le maître de cette École et ses disciples» (صسحب هذه المدرسة الفكرية وتلاميذه).

Autre difficulté selon lui, celle de l'adjectif qui précède une série de noms. Il opte dans ce cas pour des virgules après chaque mot auquel se rapporte cet adjectif. L'usage en arabe voudrait qu'on ajoute le coordonnant et après chaque substantif sans virgules. Cette répétition du coordonnant maintient non seulement une continuité discursive mais indique aussi le rôle capital de la prosodie dans la construction de la cohérence en arabe. Que fait le traducteur? Il rompt la cohérence en plaçant des virgules après le coordonnant et écrit, par exemple: «la société, et l'histoire, et les langues, et les styles, orientalistes». Cette séparation par des virgules signifiera désormais selon lui que l'adjectif se rapporte à la série des substantifs qui le précède et non au dernier seulement.

Les noms propres ne reçoivent pas le même traitement chez les deux traducteurs. Prenons comme exemple cette phrase:

Renaissance Orientalists like Erpenius and Guillaume Postel were primarily specialists in the languages of Biblical provinces [...]. (Saïd 1995: 51; notre mise en relief)

Voici ce que nous dit Abu Deeb dans sa préface concernant la transcription des noms étrangers: "j'ai tenté de les transcrire phonétiquement, à l'exception de ceux qui sont connus selon une certaine orthographe par le lecteur arabe» (Abu Deeb dans Saïd 1981 : 18; notre traduction). À l'inverse, pas de mention particulière à l'onomastique dans la préface d'Anânî.

Nous allons commenter la démarche des traducteurs à l'égard des noms propres soulignés ci-dessus. Prenons le terme de Renaissance qui en arabe se dit al nahda. Ce terme pourrait renvoyer dans l'imaginaire arabe à la période de la Renaissance arabe du XIX ${ }^{\mathrm{e}}$ siècle. C'est la raison pour laquelle Anânî choisit d'expliciter et de dire: "les Orientalistes de la Renaissance européenne», alors qu'Abu Deeb omet cette précision en se bornant à calquer la phrase anglaise: «les Orientalistes de la Renaissance». 
Erpenius est transcrit différemment chez les deux traducteurs (Erpenius chez Anânî et Erpenis chez Abu Deeb), il en est de même pour le prénom Guillaume (Ghilyaum - une transcription phonétique - chez Anânî et William - transposé selon l'orthographe arabe - chez Abu Deeb). Le substantif Bible (Biblical dans le texte) est intéressant: Abu Deeb choisit de dire la Torah (al-tawrât) tandis qu'Anânî utilise le terme approprié pour Bible en arabe et parle du Livre sacré (al kitâb al muqaddas). Ailleurs, Walter Benjamin n'est pas transcrit selon l'orthographe arabe chez Abu Deeb, contrairement à ce qu'il précise dans sa préface; il l'est par contre chez Anânî (prononcé Benyamîn).

Les noms de certaines langues, telles l'avestique ou le sanscrit, sont transcrits différemment, notamment pour l'avestique. Mais c'est surtout la présentation de ces langues qui fait l'objet d'une démarche différente. Ainsi, "the extraordinary riches of Avestan and Sanscrit» (Saïd 1995: 51) devient un calque de la structure anglaise sous la plume d'Abu Deeb, qui parle de ces langues sans préciser qu'il s'agit d'idiomes comme si le lecteur arabe connaissait nécessairement leur existence, tandis qu'Anânî parle des «trésors que recèlent des langues telles que l'avestique et le sanscrit».

L'incertitude s'étend aussi au système morphologique. En s'attaquant à la morphologie toujours dans le but de dilater la langue, Abu Deeb n'hésite pas à ébranler le style arabe, créant ainsi un sentiment d'étrangeté. Car son objectif en tant que traducteur est de transposer dans la culture d'accueil un texte dont l'univers mental est perceptible à travers sa forme. Représenter un texte, c'est en quelque sorte être fidèle au texte transposé avant d'être fidèle à la langue qui exprime cette transposition. Le choix initial du traducteur est sans conteste de se plier aux normes et à la langue du texte source. Peu lui importe la dose d'exotisation que revêtira le texte traduit, du moment que cela favorisera une certaine innovation linguistique (par exemple, la création de néologismes) ainsi que l'élargissement de l'horizon culturel du pays d'accueil.

Cette «audace» va jusqu'à forger des préfixes, faisant fi de la structuration de chaque système, structuration généralement conditionnée par les contraintes imposées par le système morpho-syntaxique qui préside à la construction lexicale. En tenir compte lors de la traduction aide énormément à la mise en place d'une cohérence structurelle. Tel n'a pas été le cas chez Abu Deeb dans les exemples suivants: ahistorical: (لَّئ- تاريخي) ; pseudo- scientific: (زي- علمي) ; et extra-academic: (زا- جامي).

Voulant imiter la troncation du terme anglais, Abu Deeb oblitère en arabe le mot censé traduire le préfixe anglais, en choisissant de sélectionner arbitrairement les deux premières consonnes du mot et qui plus est, invente un tiret entre le préfixe et la base, dans l'objectif d'imiter la monolexicalité de la langue source. Ainsi pour le préfixe pseudo, il se contente de sélectionner les consonnes «pse» pour traduire le terme et ainsi de suite. Or, la dynamique du système affixal en langue anglaise en langue étrangère n'est pas celle de l'arabe, où la tendance est à la polylexicalité. Ces créations individuelles non attestées conventionnellement sont conçues chez Abu Deeb comme des potentialités du système morphologique qu'il cherche à actualiser. Elles se comportent comme des innovations et ne valent que dans un laps de temps limité.

Le moins que l'on puisse dire est que cette façon de procéder déstabilise le lecteur arabe car elle perturbe son système linguistique et obscurcit le sens au lieu de l'éclairer, ajoutant une difficulté supplémentaire à la compréhension du texte, comme si le 
traducteur avait voulu se ménager le monopole d'accès à l'œuvre convoitée. Cette démarche est en accord avec la conception de la traduction chez Abu Deeb:

Toute traduction qui se veut ambitieuse doit viser à l'incarnation de la pensée de l'auteur dans une langue traduisante épanouie, renouvelée, apte à exprimer des concepts inédits. (Abu Deeb dans Saïd 1981: 6; notre traduction)

Bref, on l'aura compris, Abu Deeb est contre la simplification et l'explicitation, il assume ses choix en toute conscience et propose à la fin de sa préface un glossaire de termes tirés de sa traduction qu'il prend soin de définir, conscient sans doute de l'étrangeté de certains néologismes, ou, à l'inverse, heureux d'exhiber ses audaces linguistiques qui nécessitent un supplément informationnel. Il invite les traducteurs arabes à faire de même, reprochant au passage aux académiciens arabes d'établir des nomenclatures hors contexte dont l'utilité n'est pas avérée.

\section{Une réception biaisée}

Ici, l'hybridité auteur-traducteur ne passe pas inaperçue: nous sommes devant une écriture complexe, boursouflée, comme si le traducteur se regardait traduire et faisait abstraction de son lecteur, l'obligeant à se hisser à son niveau pour avoir accès au sens.

Peter Flynn (2013) s'est intéressé aux statuts et prérogatives de l'auteur et du traducteur, se demandant si le traducteur était un auteur à part entière. En effet, le débat sur les statuts respectifs de l'auteur et du traducteur rejaillit sur le degré de considération et de liberté que l'on accorde au traducteur dans son rôle de médiateur culturel. Nous avons vu qu'Abu Deeb, qui jouit d'un capital symbolique considérable, compare son sort à celui des traducteurs arabes du Moyen-Âge qui étaient eux-mêmes des philosophes ou des érudits versés dans les études théoriques et pratiques et qui, ne se contentant pas de traduire, vulgarisaient les œuvres traduites en rédigeant des introductions, des commentaires, des annotations largement repris par les philosophes eux-mêmes.

Ce faisant, ils prenaient en compte l'aptitude de leur destinataire à élargir, approfondir, ajuster sa perception de la culture de l'Autre. Ici, l'explicitation du fait culturel allait de pair avec une visée traductive particulière, concernant la totalité de l'œuvre que le traducteur entreprenait d'acclimater dans un milieu culturel arabe.

Les traductions philosophiques aboutirent, à juste titre, à la naissance d'un langage philosophique repris et amélioré par des philosophes comme Avicenne, Farâbî ou Averroès qui, ne connaissant ni le grec ni le syriaque, se fondèrent essentiellement sur les traductions pour participer au développement de ce langage philosophique. En voulant exprimer les idées véhiculées dans une langue modelée pour servir le sens qu'il entend leur donner, Abu Deeb se fait auteur et donc créateur, convaincu en son for intérieur que la langue est faite pour inventer et non pour regarder vers le passé!

Cependant, l'étrangeté des concepts proposés a biaisé la réception du livre dans le monde arabe, qui en a fait une lecture nationaliste et n'y a vu que l'opposition entre l'Orient réduit aux Arabes et à l'Islam, et l'Occident. Selon Jocelyne Dakhlia, cette lecture nationaliste a réduit le propos de Saïd à «des effets de regards croisés et de représentations en miroir [...] alors que la thèse de Saïd était justement le refus de la substantialisation des cultures et le rejet du principe même de la distance culturelle» (Dakhlia 2001 : 1180). 
L'écrivain et critique littéraire Radwa Ashur (cité dans Doushy 2013 :2) renchérit en disant qu'il s'agit d'un livre dont les thèses ont beaucoup circulé sans qu'il soit lu réellement, et que sans doute la traduction a contribué à cette situation dans la mesure où elle a réussi à transformer un livre fluide et agréable à lire en un texte difficile d'accès et alourdi par des termes obscurs.

Mais Sabry Hafez (cité dans Ali $2013: 1$ ) est plus virulent encore puisqu'il juge que non seulement les thèses brillantes de Saïd ont été altérées, mais que de surcroît la traduction a été mal perçue dans le milieu des intellectuels arabes, créant un impact négatif sur l'héritage de l'auteur. Fasciné sans doute par le style de l'auteur et voulant égaler l'original, le traducteur a pris le parti de l'étrangéité, démentant ainsi la thèse selon laquelle toute première traduction procède souvent à une naturalisation de l'œuvre étrangère et tend à réduire l'altérité de cette œuvre afin de mieux l'intégrer à une culture autre.

Le temps de la retraduction de l'œuvre de Saïd, le kairos d'Antoine Berman (1990: 5), apparaît lorsque cette retraduction devient vitale pour l'histoire d'une culture. Ce moment favorable se produisit quelque vingt années plus tard avec la traduction d'Anânî (2006).

\section{L'acclimatation de l'œuvre}

«Pourquoi retraduire?» se demande d'emblée le traducteur. Pour une question éthique tout d'abord. En effet, le lecteur arabe a pleinement le droit d'accéder au livre dans sa version augmentée de 1995, dix-sept ans après la parution de l'initiale. Dans cette nouvelle version, l'auteur Edward Saïd consacre un chapitre entier à dissiper certains malentendus et en tant que tel, ce livre intéresse la communauté arabe.

Mais ce n'est pas le seul motif selon Anânî. En effet, la langue arabe a connu une évolution fulgurante durant le quart de siècle écoulé, évolution jamais égalée explique le traducteur, même du temps de Farès Chidiaq, figure bien connue de l'époque de la Nahda, la Renaissance arabe du Xıx ${ }^{\mathrm{e}}$ siècle, et père de la modernisation de la langue arabe. En mobilisant ainsi le polygraphe du $\mathrm{XIX}^{\mathrm{e}}$ siècle, Anânî tente à son tour de donner du crédit à son argument.

La langue arabe est devenue langue officielle de l'ONU, les néologismes se sont répandus, soit par le mode de la traduction, soit par celui de l'emprunt. Le souci des académiciens a été de châtier le style, de polir la langue et de la diffuser. Les journalistes y ont contribué aussi. Le résultat a été la stabilité des termes créés, preuve indéniable de la vitalité et de l'adaptabilité de la langue arabe. Cependant, il ne faut pas croire que la création de termes soit chose facile, et le traducteur rappelle au passage les règles de la néologie et de l'harmonie phonique pour que les termes soient acceptés par la communauté des locuteurs et aient une chance de perdurer.

Cette évolution de la langue s'est également accompagnée, selon lui, d'une évolution des techniques de la traduction. Il cite au passage sa pratique traductive et les auteurs qu'il a traduits entre 1993 et 2003 (Shakespeare, Byron, Milton), tout en mentionnant deux ouvrages didactiques qu'il a écrits: "L'art de traduire» (1993) et «Les théories modernes de la traduction» (2003).

Tout ce préambule joue en faveur de la retraduction; mais au lieu de critiquer son prédécesseur et de parler de défaillance, le traducteur se contente de dire que le public arabe est en droit de lire l'héritage qu'on lui a légué dans une langue qu'il est 
capable de comprendre. Le maître mot ici est donc la compréhension, autrement dit l'actualisation du sens dans une langue qui sera comprise par les locuteurs de notre temps. Ces allusions discrètes renvoient sans les nommer à la traduction d'Abu Deeb, à ses propos sur les lacunes de la langue arabe qu'il a estimé entrevoir, aux propos des critiques et écrivains qui ont sévèrement jugé la traduction. Anânî reprend à son compte les idées et les notions de ces derniers: l'idée que l'œuvre d'un écrivain si emblématique, et a fortiori appartenant à la culture arabe, doit être accessible au public arabe, comme elle l'a été aux autres publics de par le monde. L'approche d'Anânî est celle d'un introducteur, centré sur l'horizon d'attente du lectorat cible.

Anânî livre alors sa conception de la traduction, sans chercher à ériger sa méthode en règle à suivre: elle est synonyme d'interprétation (tafsir/ijtihâd). Le traducteur explicite; et expliciter, dit-il, c'est transmettre l'idée dans une langue moderne, afin d'éviter au lecteur un risque d'ambiguiité ou d'incompréhension du sens voulu. La preuve, poursuit-il, c'est que le livre Orientalism s'est prêté à plusieurs interprétations selon les lecteurs et les générations, que ce soit en Orient ou en Occident. Il en va de même pour les traducteurs qui appréhendent différemment le livre et cela se reflète sur l'œuvre: ils peuvent compromettre sa continuité ou l'enrichir. Ainsi, pour Anânî, la lecture du texte à traduire est déjà une lecture avec interprétation, émanant de la subjectivité propre à chaque lecteur, et à chaque traducteur.

Le fait est que le style de Saïd est emblématique, qu'il puise son inspiration non seulement dans les textes littéraires mais aussi dans les textes culturels. Sa tradition intellectuelle influe sur la manière dont il expose ses arguments: adoptant une démarche spéculative puis inductive, l'auteur se reprend, rectifie des informations avant de reprendre le fil de sa pensée, multiplie les précautions d'usage. Tel est le propre d'une écriture scientifique qui a suscité des difficultés de compréhension pour le lecteur arabe, selon Anânî.

Cette entrée en matière lui permet de révéler sa stratégie traductive: dans quelle mesure, dit-il, le traducteur peut-il apporter à son lecteur des informations dissimulées dans la profondeur sémantique des textes? Selon quel dosage peut-il fournir des suppléments informationnels pour combler les lacunes culturelles supposées du destinataire de la traduction? À quoi il répond:

Ayant déjà traduit deux ouvrages de Saïd qui ont connu un succès auprès du public, je me suis familiarisé avec le style de l'auteur et j'en suis venu à penser que l'on devrait s'adresser au lecteur d'aujourd'hui et lui transmettre clairement les idées de Saïd. Pour cela, je n'ai pas hésité à introduire des ajouts explicatifs, à restructurer les phrases incises quand nécessaire, à adapter la ponctuation, à ruser parfois avec le style dans l'intention de faire comprendre. (Anânî dans Saïd 2006: 18; notre traduction)

Bref, on l'aura compris, il prend le parti de l'acclimatation de l'œuvre, de la familiarisation, contre lesquelles s'insurge Lawrence Venuti mais qui sont nécessaires à ses yeux pour que cette œuvre s'insère dans la culture d'accueil. Anânî justifie au passage les raisons qui le conduisent à rejeter la thèse de Venuti selon laquelle le traducteur doit rester fidèle aux traits culturels du texte d'origine pour gagner en authenticité (Venuti 1998). Cette thèse, selon lui, est valable pour des cultures homogènes au sein desquelles l'échange des termes et leur adoption se font avec une rapidité inouïe; il cite en exemple le terme de «discours» introduit par Foucault et que les autres langues européennes ont très vite adopté, ainsi que le terme de «différance» introduit par Derrida et qui s'est répandu à son tour. Tel n'est pas le cas des autres 
langues et cultures et notamment de l'arabe qui a sa propre manière d'exprimer les choses; par ailleurs, tout traducteur qui ne se conforme pas aux règles syntaxiques de la langue d'accueil risque de nuire à l'intelligibilité du texte traduit (Zufferey et Cartoni 2014: 364). Ainsi, Anânî préfère que le traducteur se plie aux normes du système d'accueil, pour que sa traduction soit acceptable par rapport à la langue et à la culture dudit système, au sens de Toury (1995).

De plus, la traduction chez Anânî s'apparente à l'écriture, elle doit être fluide et explicite et non obscure et étrange. En disant cela, il répond sans le citer à Abu Deeb qui, sous prétexte de "fidélité», a cherché à forger un équivalent à chaque terme au point de rendre le texte cible opaque, obligeant le récepteur à passer par l'original pour le comprendre.

Nous donnerons quelques exemples de la démarche des deux traducteurs : explicitation versus opacification. Dans le sous-titre d'un chapitre intitulé: «Orientaliser l'oriental», Anânî préfère expliciter "Conférer des attributs orientaux à l'Orient», idfâ' sifât sharqiyya 'ala al sharq, alors que Abu Deeb le formule ainsi: «Orientalisation de l'Orient», sharqanat al sharq, préférant forger un néologisme pour orientaliser.

Pour le terme de critique littéraire irony, qui se prête à la dérivation en anglais, Abu Deeb pense qu'il est difficile de cerner précisément la signification de ce terme dans les différents contextes. Ce terme ne saurait se réduire au sens qui lui est habituellement consacré en arabe et signifiant précisément ironie, sarcasme, (sukhriya), mais englobe, selon lui, la notion de paradoxe, al mufâraqa. Il propose alors le terme composé "paradoxe ironique ou humoristique», al mufâraqa al fakiha, là où Anânî s'en tient au terme courant d'ironie en arabe.

Autre remarque que l'on peut faire sur les choix terminologiques d'Abu Deeb : il inclut dans son glossaire des mots de la langue générale aux côtés des termes et les opacifie volontairement. Ainsi, le simple mot de "panorama» qui est un calque formel en arabe entré dans l'usage, est remplacé par un terme créé par l'auteur à partir de l'adjectif signifiant vaste, incommensurable, et auquel il donne une fonction de nom: shâsi'a. Anânî opte pour l'emprunt connu du lecteur arabe.

En choisissant d'inclure des mots de la langue générale dans son glossaire de 13 pages dont il donne chaque fois une définition claire, Abu Deeb veut surtout attirer l'attention du lecteur sur leur utilisation contextuelle dans le corps du texte. Hélas, le contexte ne réussit pas à dissiper l'opacité délibérée des termes, obligeant le lecteur à se référer au glossaire pour revoir leur définition.

\section{Conclusion}

La retraduction d'Anânî réhabilite une œuvre majeure et redresse considérablement les défaillances de la première: le traducteur a cherché à négocier un compromis, procédant chaque fois qu'il le sentait nécessaire à une incrémentialisation dans une visée d'explicitation. Pour cela, la langue traduisante doit être conforme au génie de la langue cible puisque à ses yeux, la traduction est tributaire des normes sociales en vigueur, elle est inséparable de la société dans laquelle elle s'inscrit et son public se définit à travers un horizon d'attente. La retraduction est par conséquent un processus légitime car lié à l'état de l'évolution de la langue et des connaissances du récepteur, mais aussi à une lecture parmi tant d'autres également possibles qu'en fait un traducteur donné. D’où son caractère dynamique et instable et une conception de la traduction proche de la réécriture, échappant à l'écueil du mimétisme, dans laquelle 
l'agentivité du traducteur s'exercerait pleinement dans l'objectif de servir l'auteur original et d'insérer l'œuvre dans son nouvel espace.

À l'inverse, Abu Deeb prend souvent le parti de l'exotisation, du décentrement de la langue cher à Meschonnic, il propose de répondre par une visibilité du traducteur, matérialisée par des concepts ne répondant pas le plus souvent aux règles néologiques de l'arabe, et par l'introduction de notes de bas de page ou de mises en relief de certains termes qu'il met entre crochets dans la version traduite et qui renvoient le plus souvent au glossaire. Le rôle du traducteur ici ne peut être réduit à celui de passeur transparent (Venuti 2002). Reconnaissons-lui cependant le mérite de s'être attelé le premier à la traduction de l'œuvre et d'avoir souligné «l'audace» d'une telle entreprise sur les plans conceptuel et linguistique. Mais si l'écriture en sciences humaines a sans doute des spécificités dans l'usage des concepts et requiert de ce fait du traducteur une cohérence terminologique particulièrement rigoureuse, traduire Edward Saïd ne se réduit pas au conceptuel. Ce qu'on vise, c'est ce que dit le texte dans la façon dont il le dit, dans la façon dont l'auteur l'a agencé. Autrement dit, un discours. Une façon, subjective, d'utiliser la langue.

\section{RÉFÉRENCES}

Ali, Hichâm (2013) : التلقي العربي لادوارد سعيد [La réception arabe d'Edward Saïd]. Al Thawra. Août 2013.

Bensimon, Paul et Coupaye, Didier, dir. (1999): Retraduire. Palimpsestes 4.

Berman, Antoine (1984): L'épreuve de l'étranger: Culture et traduction dans l'Allemagne romantique. Paris: Gallimard.

Berman, Antoine (1995): Pour une critique des traductions: John Donne. Paris: Gallimard.

Clavaron, Yves (2013) : Edward Said: L’Intifada de la culture. Coll. «Détours littéraires». Paris: Kimé.

DAKHLIA, Jocelyne (2001): «La culture nébuleuse» ou l'Islam à l'épreuve de la comparaison. Annales, Histoire, Sciences Sociales. 56(6):1177-1199.

Doushy, Amir (2013) : الترجمة مثل الكتابة فعل انتساب واثبات للذات [La traduction tout comme la réécriture est un acte d'affiliation et d'affirmation de soi]. Al Sabah. Juillet 2013.

Flynn, Peter (2013): Author and Translator. In: Yves Gambier et Luc van Doorslaer. Handbook of Translation Studies. Volume 4. Amsterdam/Philadelphie: John Benjamins, 12-19.

Genette, Gérard (1987): Seuils. Paris: Seuil.

Inghilleri, Moira (2003): Habitus, Field, and Discourse: Interpreting as a Socially Situated Activity. Target. 15(2):243-268.

IsRAËL, Fortunato (1994): La créativité en traduction ou le texte réinventé. In: Margit RADERS et Rafael Martín-Gaitero, dir. IV Encuentros Complutenses en torno a la Traducción. Madrid: Editorial Complutense, 105-110.

Latour, Bruno (1987): Sciences in Action. Cambridge: Harvard University Press.

MejRi, Salah (2008): La traduction des textes spécialisés: le cas des sciences du langage. In: Christian Balliu. Traduire: un métier d'avenir. Volume 2. (Colloque du 50 anniversaire de l'ISTI, Bruxelles, 14-15 octobre 2008). Bruxelles: Les Éditions du Hazard, 117-144.

Moura, Jean-Marc (1999): Littératures francophones et théorie postcoloniale, Paris: Presses universitaires de France.

SAÏD, Edward W. (1978/2006) : المفاهيم الغربية للثرق [L'orientalisme: l'Orient, au regard de l'Occident]. Traduit de l'anglais par Mohamed AnÂnî. Le Caire: Ru'yâ.

SAÏD, Edward W. (1978/2003): Orientalism. London: Routledge \& Kegan Paul Ltd.

SAÏD, Edward W. (1978/1981): الاستشر اق: الإنشاء، السلطة، المعرفة :L'orientalisme: le discours, le pouvoir, le savoir]. Traduit de l'anglais par Kamal Ави DeEв. Beyrouth: Institut arabe des recherches. 
SAÏD, Edward W. (1978/1980): L'orientalisme: L'Orient créé par l'Occident. Traduit de l'anglais par Catherine Malamoud. Paris: Seuil.

SAmain, Didier (2014): Entropie et néguentropie de la traduction. Revue suisse des sciences de l'éducation. 36(2):179-194.

Shahine, Mohamed (2007) : أسفار في عالم الثقافة [Voyages dans le monde de la culture]. Beyrouth: Institut arabe des études et de l'édition.

Toury, Gideon (1995): Descriptive Translation Studies and Beyond, Amsterdam/Philadelphie: John Benjamins.

Vendryes, Joseph (1921/1968): Le langage, introduction linguistique à l'histoire. Paris: Albin Michel.

Venuti, Laurence (1998): The Scandals of Translation. Londres/New York: Routledge.

Venuti, Laurence (2002): The Translator's Invisibility. Londres/New York: Routledge.

Zufferey, Sandrine et Cartoni, Bruno (2014): A multifactorial analysis of explicitation in translation. Target. 26(3):361-384. 